\title{
Digital Form Finding Using Voronoi Pattern
}

\section{Mara Capone $^{1}$ (D) . Emanuela Lanzara ${ }^{1}$ (D) . Francesco Paolo Antonio Portioli ${ }^{1}$ (D) . Francesco Flore ${ }^{1}$ (D)}

Accepted: 18 June 2021 / Published online: 9 July 2021

(c) The Author(s) 2021

\begin{abstract}
Starting from funicular models, chain models and hanging membranes, the role of $3 \mathrm{D}$ physical models in optimized shape research is the basis of form-finding strategies. Advances in structural optimized shape design derive from the wide spread of special digital form-finding tools. The goal of this paper is to test and evaluate interdisciplinary approaches based on computational tools useful in the form finding of efficient structural systems. This work is aimed at designing an inverse hanging shape subdivided into polygonal voussoirs (Voronoi patterns) by relaxing a planar discrete and elastic system, loaded at each point and anchored along its boundary. The workflow involves shaping, discretization (from preshaped paneling to digital stereotomy) and structural analysis carried out using two modeling approaches, finite element and rigid block modeling, using an in-house software tool, LiABlock_3D $\left(\right.$ MATLAB $\left.^{\circledR}\right)$, to check the stress state and to evaluate the equilibrium stability of the final shell.
\end{abstract}

Keywords Digital form finding $\cdot$ Voronoi pattern $\cdot$ Masonry domes

Mara Capone

mara.capone@unina.it

Emanuela Lanzara

emanuela.lanzara@unina.it

Francesco Paolo Antonio Portioli

francescopaoloantonio.portioli@unina.it

Francesco Flore

francescoflore@desvamiz.com

1 Department of Architecture (DiARC), Università Degli Studi di Napoli Federico II, Naples, Italy 


\section{Introduction: Computational Tools to Simulate Form Finding Approaches}

The role of the 3D physical model in optimized shape research is the base of formfinding strategies. The 3D model is always used to simulate processes, and to define optimized complex shapes. Starting from Gaudí's funicular models, Frei Otto's chain models and reversed Isler's hanging membranes, advances in structurally optimized shape design derive from the widespread availability of digital form-finding tools that make it possible to test several research directions. Through an interdisciplinary collaboration between computer science and architecture, and architects and engineers, the goal of this paper is to test and evaluate different approaches based on computational tools useful for efficient form finding in the design of 3D structural systems by means of an iterative process (Henrique et al. 2020: 171) (Fig. 1).

This research work is aimed at designing an inverse hanging shape subdivided in polygonal voussoirs (using Voronoi pattern) by relaxing a planar discrete and elastic system, loaded in each point and anchored along its boundary. The workflow involves three main steps: shaping, discretization (from paneling to digital stereotomy), and structural analysis. Modeling and discretization are managed according to a Visual Programming Language (VPL) algorithmic generative approach (Grasshopper, Rhino) using a specific add-on to simulate forces and anchoring conditions and to model voussoirs (Rippmann et al. 2011b: 183) starting from Voronoi cells (mesh). These models are dynamic systems (Sulpizio et al. 2020: 30) that can be modified in real-time by changing special parameters: geometric pattern, boundary conditions, physical forces, anchor system, loads, materials, stress state.

In this preliminary step, the structural behavior is simulated according to a special digital tool, the live physics engine Kangaroo (see below), which is generally based on mass-spring models. In the third step, a detailed structural analysis is carried out using two types of modeling approaches to check the stress state and to evaluate the equilibrium stability of the shell configuration obtained from the shaping and discretization steps (Fig. 2). The first modeling approach is the finite element method (FEM) implemented in the software suite Abaqus FEA, while the second approach is

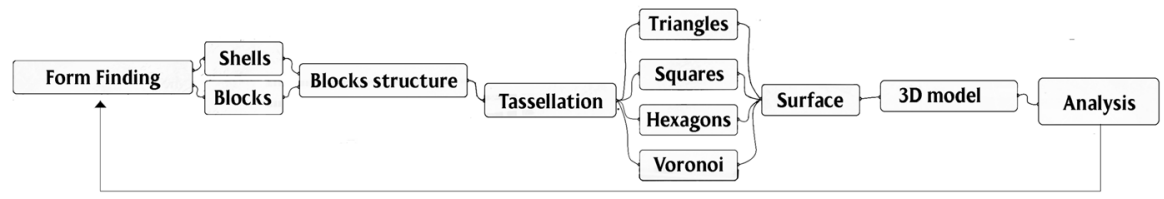

Fig. 1 Digital form finding: iterative process

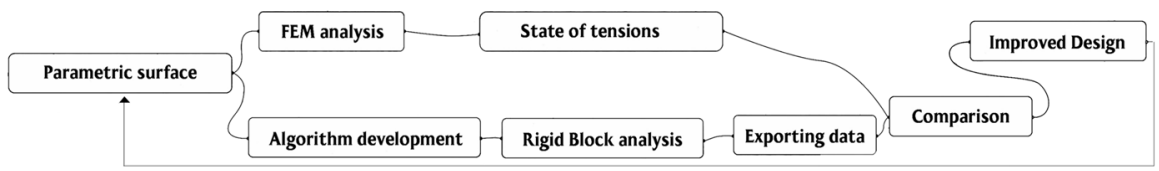

Fig. 2 Iterative workflow of structural analysis 
rigid block (RB) modeling. This second approach was used to evaluate the structural safety against potential local failure mechanisms according to an in-house software tool (named LiABlock_3D), which was developed in MATLAB ${ }^{\circledR}$ (Cascini et al. 2020: 75-94). The code was generated within a research project which is aimed at developing efficient and reliable computational tools based on mathematical programming for the assessment of block structures by nonlinear time-history, static pushover, and limit equilibrium analysis (Portioli 2020: 211-239).

Traditional architectural shapes are based on geometric rules such as revolution or translation of surfaces, lofting, surfaces intersection, curves interpolation, Boolean transformation, and so on. The 3D modeling technique used to represent the traditional shapes does not allow to define an optimize solution, unlike the formfinding approach.

Instead, the growing complexity of today's architectural design process requires approaches aimed at optimizing processes, minimizing available resources. Curved and complex shapes make it possible to test and to optimize compositional and technological solutions aimed at creating more sustainable architectures. Nowadays, structural analysis using user-friendly digital tools is more accessible in the design phase, although information and data critical interpretation depends on engineering theoretical prerequisites. Special digital tools based on a VPL approach allow designers to independently experiment with funicular systems also in absence of professional expert knowledge (Rippmann 2016: 9). Therefore, the main goal of this approach is to manage the creative process, guaranteeing its feasibility and sustainability. In particular, the main challenge consists in combining shaping with more suitable paneling systems to ensure optimal environmental, structural, economic, and aesthetic performance. According to the digital form-finding approach, parametric generative tools allow designers to explore different shapes by merging the creative phase and structural analysis: the structure defines its own shape according to the equilibrium condition, then the configuration, of a given system under the action of loads.

From Gaudí's catenaries to Heinz Isler's hanging membranes, shapes were realized and tested using material prototypes. The increasing development of special parametric-computational tools allows researchers and designers to simulate the same form-finding approach in the digital environment, optimizing the whole process (Fig. 3). Testing, modifying and validating these configurations in real-time without the onerous manufacturing and verification of material models favors the use of these tools. In addition, digital form finding processes allow global surface deformation to generate optimized shapes characterized by gradual curvature variations, favoring continuity and regularity of shapes (Capone et al. 2014). This approach facilitates the genesis of mechanically optimized complex geometries without resorting to other modeling or post-shaping editing techniques that can undermine system feasibility due to sudden changes in curvature and irregularities (Liu et al. 2006).

Therefore, the designed hanging membranes were transformed into a corresponding cable net by testing different patterns, regular (triangular, quadrangular and hexagonal), and irregular (Voronoi). Pattern, block shape and distributive layout decisively contribute to the performance of the entire system. 

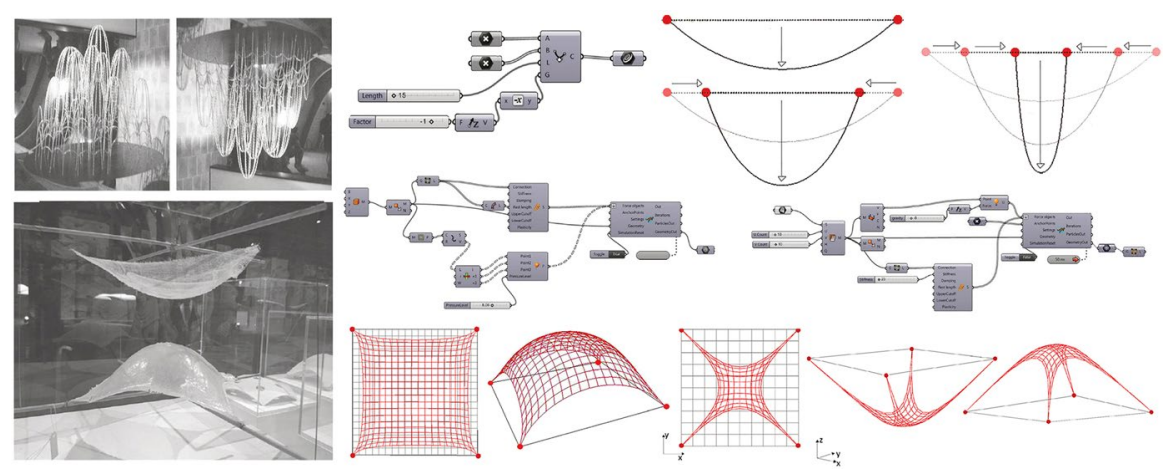

Fig. 3 VPL algorithmic generative tools for digital form finding. Special tools (on the right) allow to simulate Gaudí's catenaries and Isler's membranes form finding approach (on the left)

This process also allows a possible stereotomic approach for manufacturing complex shapes.

\section{Form Finding: From 2D Boundary to 3D Model}

Here we illustrate a digital form-finding process aimed at finding an optimized complex shape designed to cover irregular regions. History provides many examples of using hanging and reverse shapes to design architectural elements employing physical models to obtain structures resistant to compression. Michelangelo (1475-1564), as architect and engineer, reduced the weight of the structure using the method of double skin shell, suggested by Filippo Brunelleschi (1377-1446). Later, in 1580, Giambattista Della Porta (1535-1615) added the height-to-span ratio of the domes by extending their lower parts. Military and civil engineer Simon Stevin (1548-1620) was one of the first to develop the mathematical representation of forces as vectors in his book De Beghinselen der Weeghconst, published in 1586. He illustrated the parallelogram of forces and showed several examples of suspended weights creating funicular shapes in both two and three dimensions. Christopher Wren (1632-1723) and Robert Hooke (1635-1703) worked on the design of a $33 \mathrm{~m}$ diameter dome of Saint Paul's Cathedral in London using the suspended chain method and Giovanni Poleni (1683-1761) employed Hooke's method in the evaluation of the $50 \mathrm{~m}$ diameter dome of St. Peter's in Rome (Adriaenssens et al. 2014: 35). Friedrich Gosling (1837-1899) used two- and three-dimensional arches in his models in the 1890s. The method was further developed by Antoni Gaudí (1852-1926), who employed small weights suspended from strings to realize models of masonry shells. Frei Otto (1925-2015) used material models to determine the tension in cable networks when a digital approach was unfeasible. Finally, Heinz Isler (1926-2009) designed and built several complex and stable shells inspired by Hooke's method (Timoshenko and Woinowsky-Krieger 1959) starting from simple geometries of reference, according to design or environmental factors. 

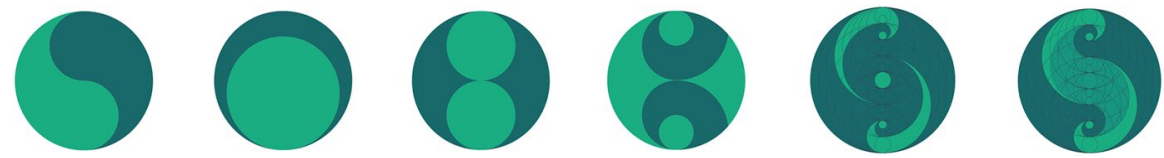

Fig. 4 Searching for a boundary of reference: circle subdivisions

Contemporary complex architectural shapes cannot be realized according to exact calculations without the use of special tools and of specific knowledges and skills. Mimicking natural shapes allows designers to find optimized complex shapes without intensive calculations (Castro et al. 2020). Therefore, due to the complexity of shell geometries, the historical trials were usually restricted to exactly calculating simple geometries, such as the shapes proposed by Timoshenko and WoinowskyKrieger (1989). Isler's exceptional ability to model shells according to simple configurations inspired many designers interesting in the field of form finding (Baghdadi et al. 2019: 492-494). This approach provides shaping by hanging and finding simple forms of reference according to the effect of gravity to achieve optimized systems under pure tension (compression), independent of their size, and attaining optimal and economic results. This means that making and testing small physical models can be a quick and practical method. Even major contemporary researchers, such as BRG (Block Research Group, block.arch.ethz.ch/) and Alberto Pugnale (albertopugnale.com/) adopt this logic in designing structurally optimized forms and testing these approaches.

According to all these premises, the boundary of the planar shape that we have chosen for our first tests is a polycentric curve, deriving from the discretization of a circle (Fig. 4).

In this context, we are going to underline that the planar shape of reference is a result of a design process in which the designer has to define the plan in relation to specific needs. In our case, the final plan derives from the geometric decomposition of a circle and the distribution of strategic openings to guarantee visual permeability, internal/external interaction, and distribution of any internal functional spaces. However, irregular profiles generate curvature variations in 3D membranes by relaxing meshes starting from specific boundary anchoring conditions. The structural behavior of a shell also varies according to the pattern chosen to discretize the planar region of reference (mesh) and the location of the anchor points. By fixing the force weight value and varying the stiffness parameter of springs (Springs component, Kangaroo), the number of isoparametric curves $u$ and $v$ related to the cells number (Panels components $>$ triangle panels, quad panels and hexagon cells, LunchBox) or the number and distribution of Voronoi cells (Mesh components $>$ Voronoi 3D $>$ Points), it is possible to observe morphological variations during the simulation. Stiffness value and relaxation/ deformation are inversely proportional: the designer guides the process to control output based on variations in these parameters. By increasing the number of isoparametric curves and setting the stiffness parameter, it is possible to obtain greater deformation deriving from the original flat mesh refinement. In this case, 


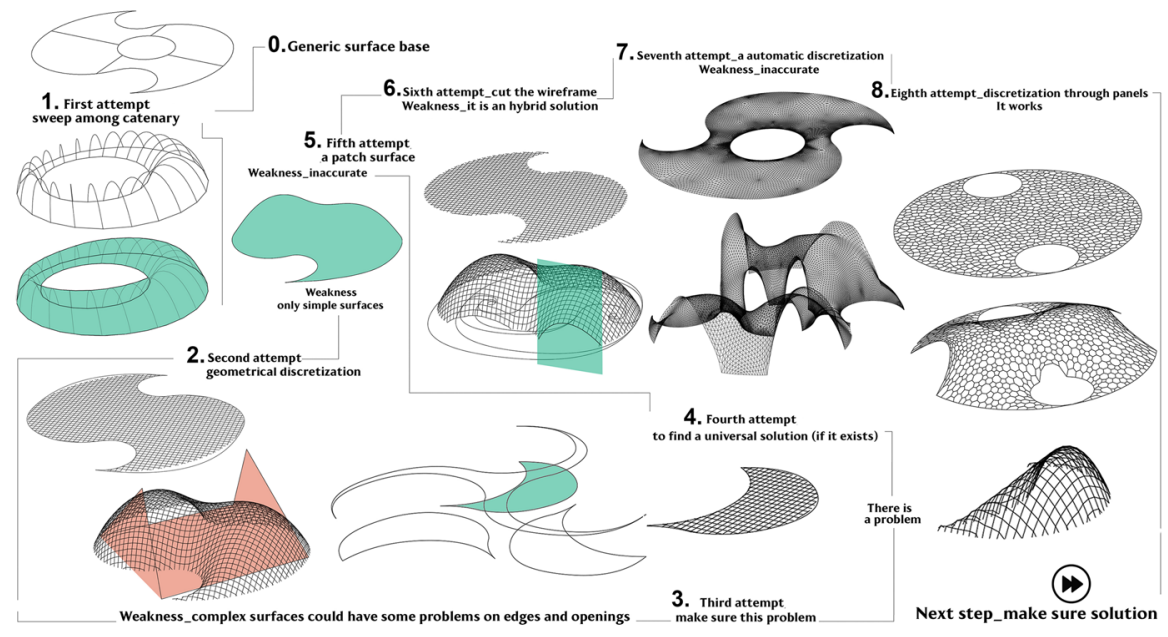

Fig. 5 Searching for the boundary of reference according to optimized digital form finding

to contain deformation it is possible to increase stiffness value. Therefore, the aim of our research is to test different boundaries and patterns and to compare their structural performances to define the best shaping and paneling strategies to use for the design shape (Fig. 5).

\section{Digital Form Finding and Paneling}

Our research illustrates a set of digital form-finding tests to search for favorable geometric conditions aimed at combining formal identity with complex selfsupporting surfaces. Computing and verifying design solutions according to a pre-rationalization approach (Shiftner et al. 2012: 216-220) favors dynamic and performing architectural systems. This approach simulates physical forces applied to points and curves that discretize the region of reference: deformation generates different configurations. The digital form-finding simulation makes it possible to interactively manipulate shapes and to visualize Gaussian curvature variations of each configuration in real time, especially for convex configurations that mostly work under compressive stresses. The form-finding approach developed by Isler to manufacture thin hanging and then inverted membranes consists of immersing a fabric anchored at its vertices in a mixture of water and plaster. The relaxed system solidifies by contact with air and the resulting shape can be turned upside down. The form of relaxed membranes depends on the initial planar grid, the boundary and the position of anchor points. The open-source digital tools used for simulating and testing this approach are Rhino and its Grasshopper plug-in, 


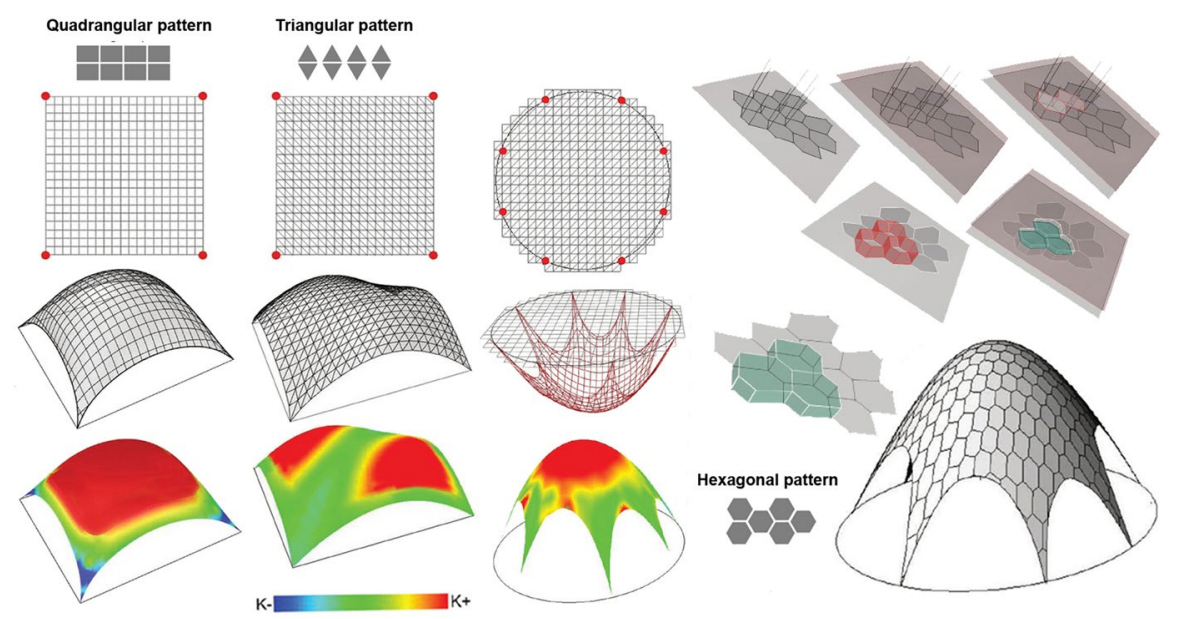

Fig. 6 Post-shaping paneling approach. Tessellation after digital form finding (Lanzara 2019: 221-227)

combined with its add-ons Kangaroo, ${ }^{1}$ LunchBox, ${ }^{2}$ Weaverbird ${ }^{3}$ and NGon. ${ }^{4}$ Kangaroo is a live psychics engine to digitally simulate physical forces (force vectors), including the components Unary Forces (strength weight), Catenary (catenaries) and pressure (pneumatic system, compressible gas) acting on points of a discrete input system. The engine also allows the designer to planarize cells (Planarize component) to optimize paneling manufacturing. LunchBox and Weaverbird are special add-ons that make it possible to tessellate surfaces and manipulate meshes. Force weight simulation is enabled by Kangaroo's Unary Force component, which allows us to transform a flat and continuous NURBS surface first into a mesh, and then into a discrete relaxed system physically and structurally like a real fabric lattice. The shaping and paneling steps can be addressed simultaneously or separately. Hence, we have identified two different approaches: post-shaping and pre-shaping paneling. In the post-shaping paneling process, we use a generic grid to divide the irregular planar region, we relax it, and we generate the design surface (mesh). We finally paneling the design surface according to the chosen pattern. Tessellation and block generation independent of the original grid is possible by linking a continuous geometric representation to the thrust network (Lachauer et al. 2010: 3). In contrast, in the pre-shaping paneling approach we use the same pattern to obtain the planar region discretization and the paneling design surface. In this way the mesh of reference and the paneling system will be the same. The experimentation shown in this contribution is based on pre-shaping paneling (Fig. 6).

\footnotetext{
1 Kangaroo physics by Daniel Piker, https://www.food4rhino.com/app/kangaroo-physics.

2 LunchBox by Nathan Miller, https://www.grasshopper3d.com/group/lunchbox.

3 Weaverbird-Topological Mesh Editor by Giulio Piacentino, http://www.giuliopiacentino.com/weave rbird/.

${ }^{4}$ Ngon by petrasvestartas, https://www.food4rhino.com/app/ngon.
} 


\section{Hanging Membranes and Voronoi Pattern for Optimized Shell}

Let's consider the region of reference to extract the planar mesh according to several (regular and irregular) patterns. By relaxing it, we will achieve the final shape that is quite the same as the hanging membrane. Therefore, the first problem is to discretize a flat region delimited by a curved and complex boundary with a regular or irregular pattern. However, the choice of a grid that is regular (triangles, quadrilaterals, hexagons) or irregular (Voronoi) also depends on structural performance. Structural performances of each pattern are verified by the FEM applied to the discrete shell. As stated, convex shells mainly work at compressive stresses (Sasaki 2005). Therefore, to achieve convex shells, virtual weight forces are applied on internal vertices of polygonal cells, and the mesh is constrained at anchor points selected along mesh boundary according to specific design goals. Furthermore, the position of the anchor points also influences the shape curvature. In the case of a planar region with a curved profile, the available digital tools approximate it in a modular grid, recalling the method of exhaustion, a mathematical procedure for calculating areas of planar geometric figures that consists of the construction of a succession of polygons converging to a given figure. Thinking about the shape of a planar grid of reference offers an interesting stimulus to test many different possible solutions. Studies and experiments show that surfaces with a predominantly positive Gaussian curvature allow the distribution of regular flat-convex hexagonal panels.

Hexagonal panel planarization is mainly aimed at finding optimized economic, aesthetic, and constructive solutions to manufacture complex shapes. However, in the architectural field, this pattern is still little used despite its numerous advantages, such as its offset properties useful for creating multilayer systems to promote energy and structural performance of envelopes and facades, and to develop digital stereotomy applications.

Next, the resulting curved region is meshed according to a given number of isoparametric curves $u$ and $v$, defining its subdivision in both directions. The mesh structure allows us to geometrically simulate fibers of a real elastic membrane. We are transforming the hanging membrane into a corresponding cable net.

Meshes based on regular (triangular, quadrangular, hexagonal) or irregular (Voronoi) patterns are exploded into vertices and curves and transformed into an elastic system (Springs component, Grasshopper, Rhino). Transforming geometric elements into "ideal springs" allows controlling a series of parameters, including stiffness or plasticity of material assumed for simulation, by setting adequate values to prevent a membrane from being "infinitely" relaxed or too rigid. Force is simulated by applying parallel z-axis vectors on each point of the achieved discrete and elastic system: unary forces are applied to each of the points, using oriented vectors. The planar mesh of reference belongs to the $x y$ plane, therefore the vectors representing weight force will be directed downwards. The gravity force action simulation on mesh vertices requires an anchoring system (anchor points) to avoid detachment of the relaxed membrane from tge support. The first tests foresee membrane anchoring along the entire boundary (attempting shape), testing different patterns (triangular, quadrangular, hexagonal and Voronoi), and comparing the results: according to the same parameters, the quadrilateral mesh, unlike the more 


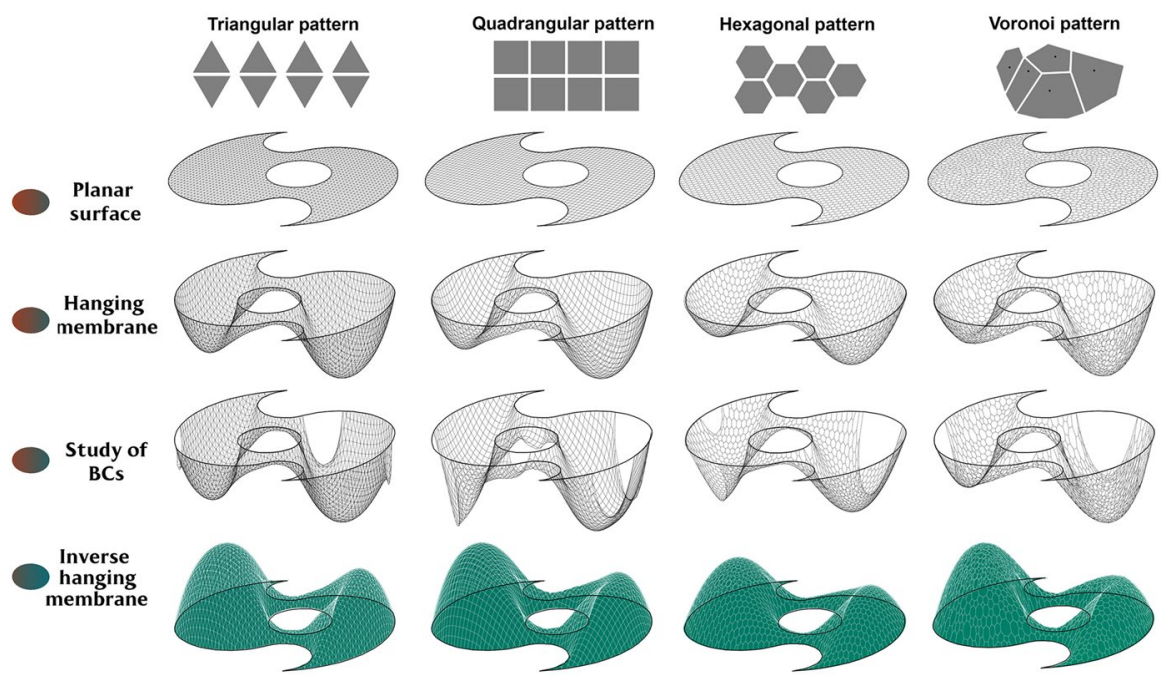

Fig. 7 Pre-shaping paneling approach: testing pattern for digital form finding according to design goals

rigid triangular mesh, allows a more "homogeneous" relaxation, corresponding to a more uniform and almost totally positive Gaussian curvature distribution (Fig. 7).

Our tests show that the Voronoi pattern is performs better in terms of membrane relaxation and structural performance. A Voronoi tessellation is a subdivision of a plane into cells based on the distance to points in its specific subset. That set of points (called seeds, sites, or generators) is specified beforehand, but for our test, a random set of points was used. Therefore, the Voronoi structure starts from a random distribution of points on the planar region of reference. These points represent vertices of Voronoi cells that populate a closed region. From these points, which are the center of our shape, circles were generated. Their intersections produce the same number of polygons with a different number of edges. Cells along the boundary are open: therefore, it is necessary to transform them into closed polylines, then exploding the meshes to separately select vertices (unary forces application) and edges (springs).

Figure 8 shows the progressive evolution of the final boundary. The covered region is equal to $245 \mathrm{~m}^{2}$, while the whole circular area is equal to $490 \mathrm{~m}^{2}$ and the number of Voronoi cells is equal to 200.

Geometry (points, linear connections), forces (unary forces and springs), and anchor points are the input parameters for Kangaroo. The perturbed particles reach equilibrium and flat elastic meshes relax. To distribute arches in the final shell, groups of points distributed along the boundary of the region were excluded. Then, at those points, the membrane is released. The position of arches (height and shape are calibrated through relaxation) were controlled by building a series of optical cones whose vertices are distributed inside the shell to simulate strategic viewpoints to allow system permeability. This approach is aimed at generating a structurally optimized configuration meeting specific functional needs (Fig. 9). 

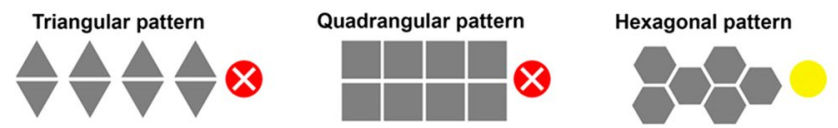

Voronoi pattern
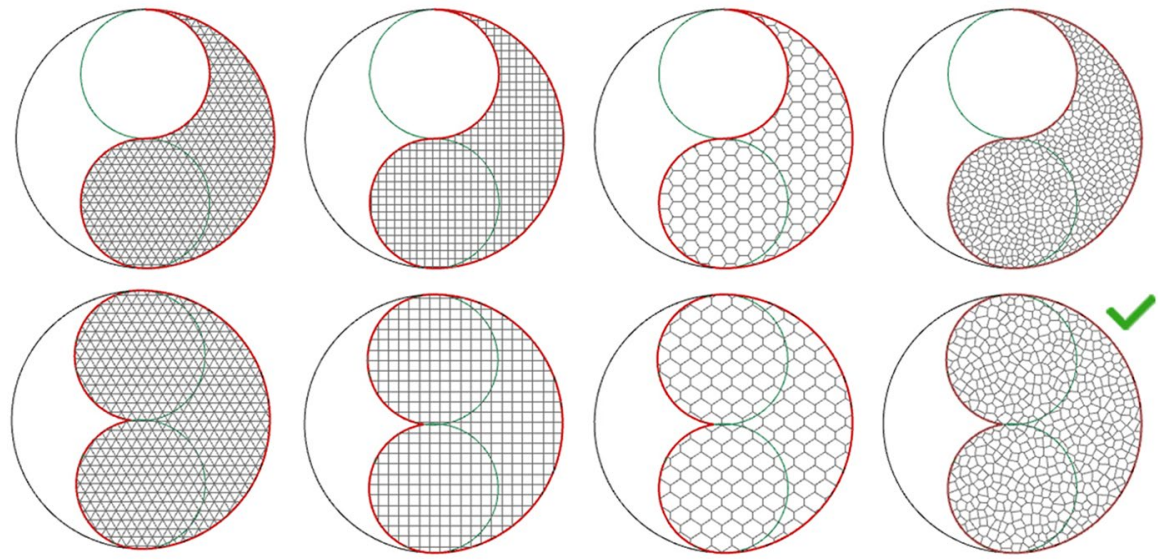

Fig. 8 Boundary evolution. Pattern distribution on the planar region of reference

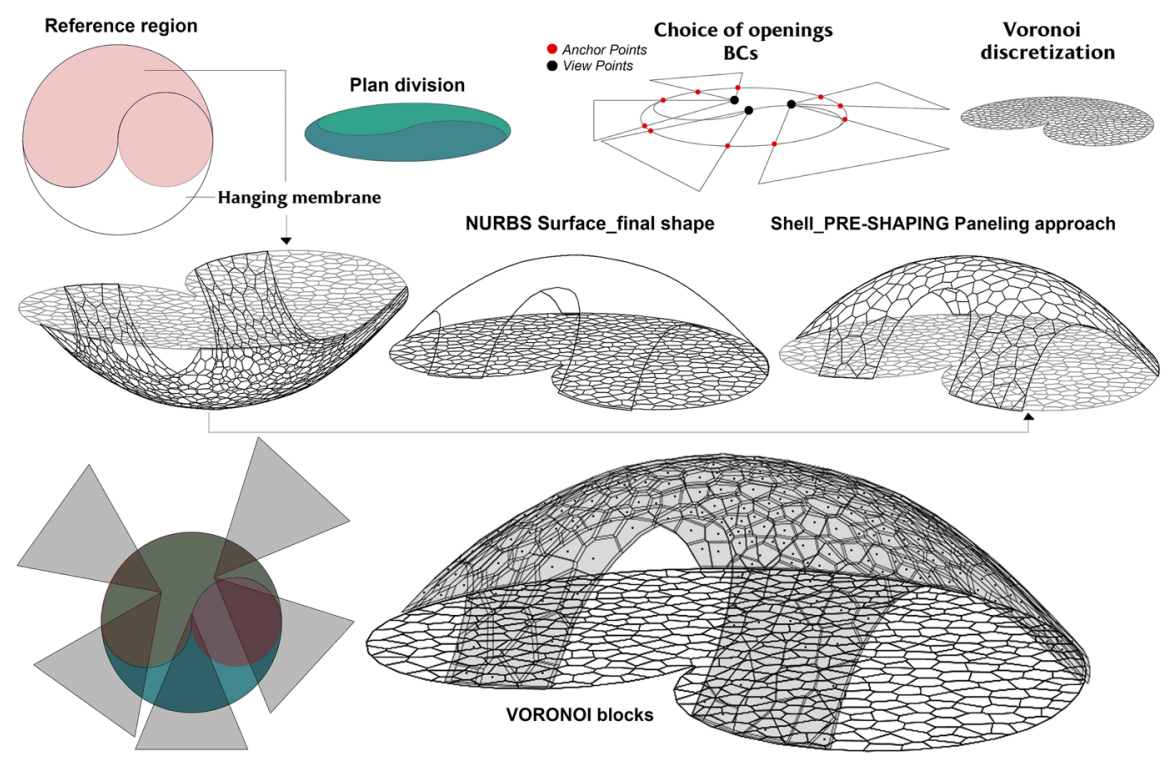

Fig. 9 Final solution: structurally optimized configuration and paneling solution meeting specific functional needs 


\section{From Paneling to Digital Stereotomy}

In our research we are testing a design process for the digital manufacturing of an optimized surface using Voronoi blocks. We had to deal with two main questions: the geometric question linked to stereotomy and the structural question. The main topic is how to manufacture a continuous surface that looks like a thin shell structure using blocks.

Offset discrete systems composed of flat polygonal elements allow transformation of cells into volumetric adjacent elements. The faces of these elements can be flat or curved. This advantage allows to evaluate a possible stereotomic approach to manufacture complex shapes.

A stereotomic system, according to the number and shape of elements, can return the curvature of a continuous system of reference without high approximation. Stereotomy is the science of structures of masonry or other materials whose elements are made according to careful prefabrication processes. A stereotomic system subsists in its form by the geometries of its parts, which must be prefabricated to dry-build an organic architectural ensemble. Basically, this science studies projective rules to design shapes aimed at being cut so as to realize complex architectural systems (Fallacara 2007: 36).

It is possible to define three stereotomic principles: prefigurative invariant, to divide a system into parts; technical/geometric invariant, to describe the geometry of a system and its parts; static invariant, to ensure the static balance of the architectural system by dry assembly of its segments (Salvatore 2009: 486). According to these principles, stereotomic design studies the subdivision of a continuous system: the geometry of each element is inextricably dependent on the nature and transformations of the entire system. Thus, the single part strongly depends on the whole configuration. According to these premises, it is interesting to apply these principles to investigate alternative solutions for complex shapes manufacturing.

In offsetting a discrete system resulting from a complex surface, each voussoir will necessarily be different from the others. This technique requires a theoretical treatment starting from principles described in stereotomy treatises both ancient and contemporary (Fallacara 2007; Salvatore 2012). Hence, the relationship between computational design and digital fabrication sets up stereotomy as a very topical science. The main steps (Rippmann et al. 2011a) of the current stereotomy approach are: form finding (shaping), tessellation, segment cutting (voussoirs; see Rippmann and Block 2011b: 183) and assembly. The Block research Group of the ETH Zurich, mentioned earlier, whose research aims to develop methods for the tessellation of complex vaulted systems by the distribution of solid blocks, and the Institute for Computational Design and Construction of the University of Stuttgart emerge among leading research groups engaged in the development of digital stereotomy approaches. Offset-mesh testing demonstrates the utility of the investigation and systematization of the basic theoretical principles of digital stereotomy: stereotomic design inevitably requires knowledge of the geometric rules for controlling 


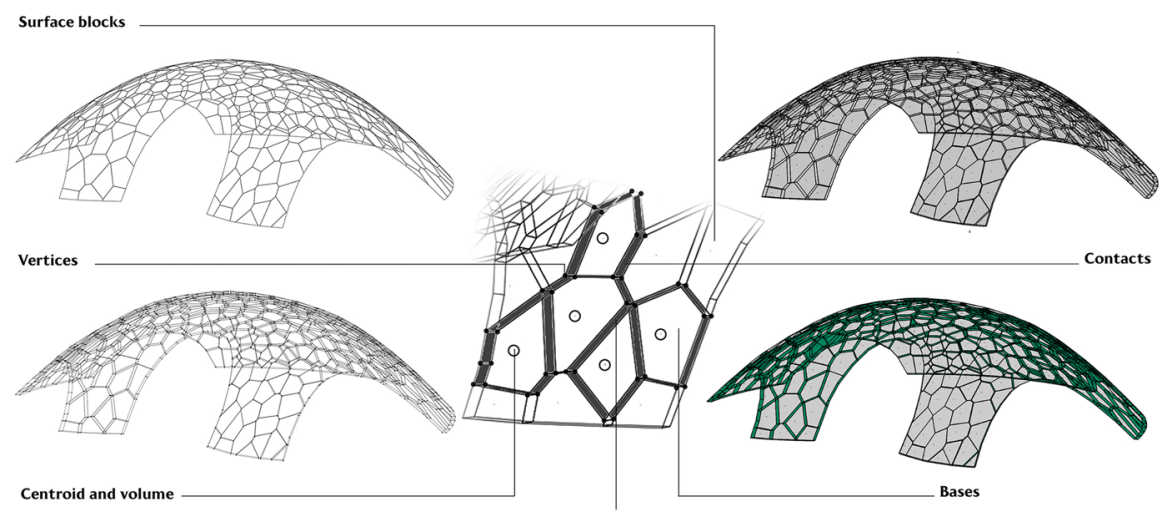

Fig. 10 Distribution and detail of adjacent Voronoi blocks, showing contact faces, edges and vertices

the overall morphology of an entire architectural organism and of its parts. Therefore, it is interesting to combine advanced knowledge of complex surfaces and contemporary rationalization techniques with basic knowledge of the science of stereotomy. Figure 10 shows the distribution and a detail of adjacent Voronoi blocks, showing contact faces, edges and vertices. Section 6 presents a discussion of the calculation of the thickness of segments to guarantee the best structural performance.

\section{Structural Analysis}

For structural analysis, FEM and RB analyses were carried out to verify the stress distribution and the formation of failure mechanisms when the structure is subjected to load configurations which might be different from those used for the shape optimization study, such as the varying distribution of live loads. In this case, we assumed that the structure is made of stone blocks with a compression strength equal to 20.0 MPa and a tensile strength of 1.0 MPa. For variable loads, the effects of snow loads were considered according to Eurocode 1 (CEN 2003), assuming a total design load of $9 \mathrm{kN} / \mathrm{m}^{2}$ including the effects of self-weight. The FEM model was generated in Abaqus, importing the surface which was obtained from the form-finding study. In this case, a static analysis was carried out adopting an elastic material model (with the Young modulus and Poisson ratio equal to 0.3 , respectively). S4R shell elements were used for meshing. The objective of the finite element analysis was to design the thickness of the block vault in order to meet stress verifications. The results of the FEM analysis in terms of minimum and maximum principal stresses (expressed in $\mathrm{MPa}$ ) are shown in Fig. 11, for a thickness equal to $16 \mathrm{~cm}$.

In the case of RB analysis, the structural safety was assessed in terms of collapse loads and related failure mechanisms. The structure is idealized into an assemblage of rigid blocks interacting at no-tension, frictional contact interfaces. 


\section{FEM analysis}

Stress distribution under gravity loads for tensile strength control
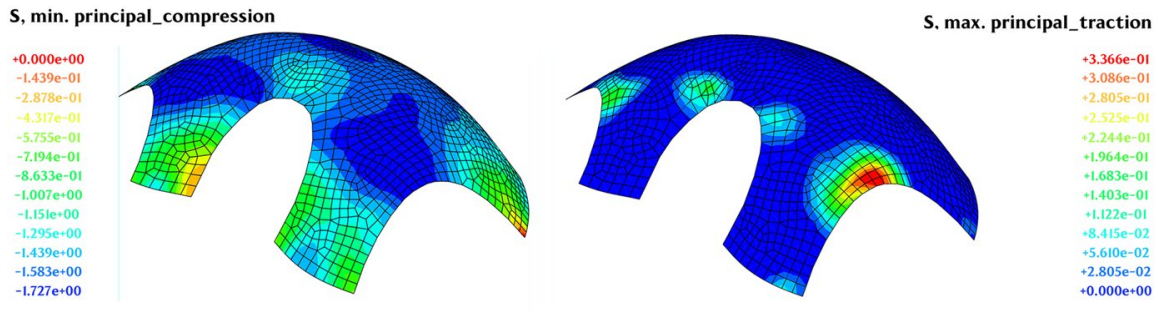

S, mises_tensions
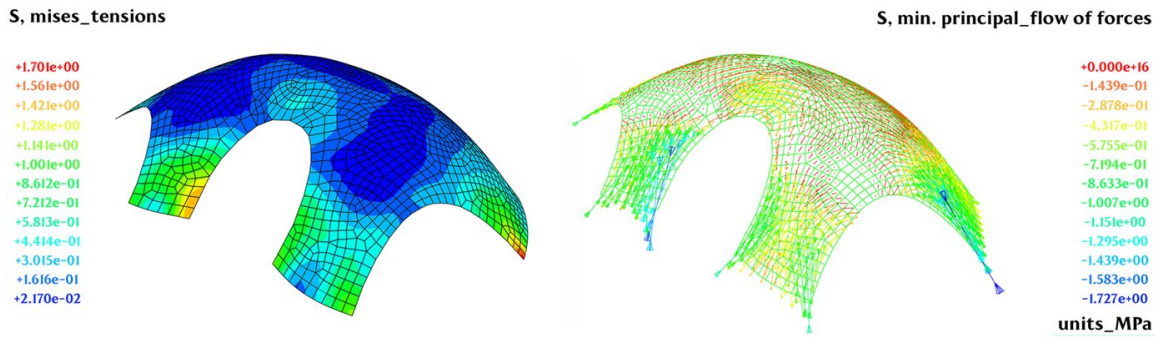

Fig. 11 FEM analysis. Stress distribution under gravity loads for tensile strength control

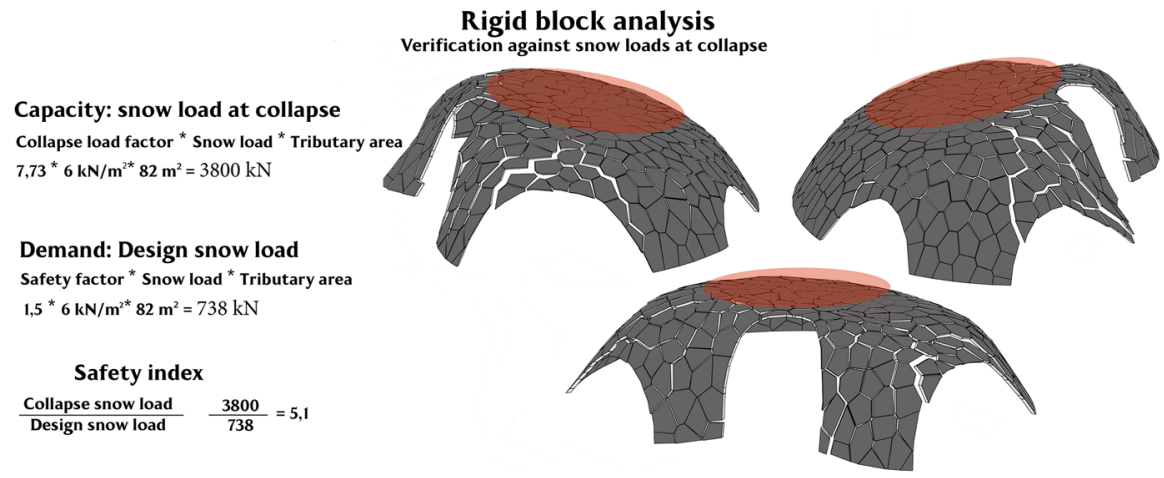

Fig. 12 Rigid block analysis. Verification against snow loads at collapse

The limit equilibrium problem corresponding to a failure mechanism is posed as a mathematical programming problem, namely as a second order cone programming problem, following the classic static approaches of limit analysis. The objective function is the collapse load multiplier of variable loads, while the constraints of the optimization problem are the equilibrium conditions at each block and failure conditions at contact interfaces (Cascini et al 2020: 75-94). The results of the rigid block analysis with the LiABlock_3D code are shown in Fig. 12.

A procedure for extrapolation and export of geometric data was implemented for each structural component. By assigning thicknesses and pattern to the 


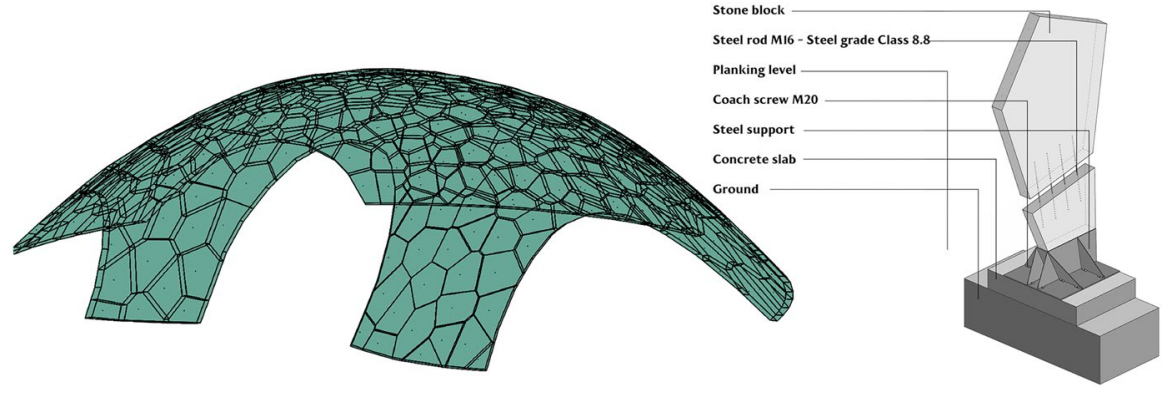

Fig. 13 Final optimized discrete shell and ground anchor detail

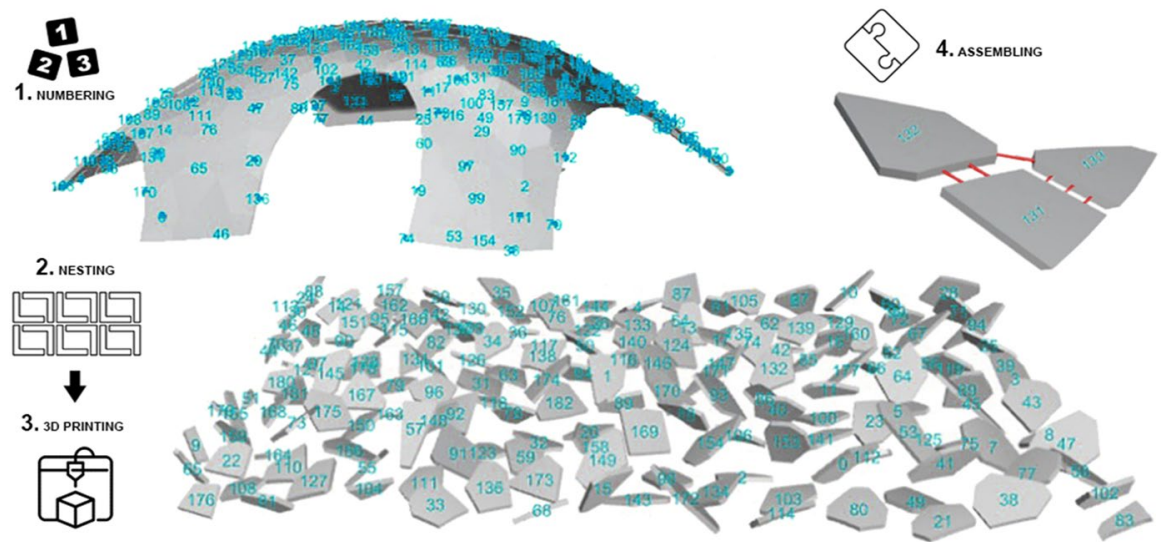

Fig. $143 \mathrm{D}$ blocks numbering, nesting and assembling hypothesis. The number of bars depends on the width of the contact area between the blocks

meshes (LunchBox, Weaverbird, NGon) and the center of gravity, the volume of contact surfaces and nodes to each block, it is possible to process and translate resulting data in an electronic spreadsheet. Therefore, we developed a VPL algorithm (Grasshopper, Rhino) to export the necessary data. In this way, we calculated the collapse factor for snow loads, that is, the snow load that induces the activation of a failure mechanism. The safety index, that is, the ratio between snow collapse load and the design load according to Eurocode 1 (CEN 2003), is equal to 3.4. For design purposes and in order to entirely prevent potential sliding phenomena, steel bars were also considered at block interfaces. According to these analyses, $20.0 \mathrm{~cm}$ of thickness represents the best solution. Finally, this thickness was assigned to the shell using the Offset Mesh component (NGon add-on) to complete a digital 3D model of the shell (Fig. 13). 


\section{Conclusions}

Our interdisciplinary research is a work in progress that allows us to compare different points of view. The main goal is to test new design processes to improve the use of algorithmic generative modeling tools able to define a structurally optimized shape. In this case, modeling allows designers to simulate structural behavior and to look for the best solution using a generative approach. Experimental investigations should be carried out to validate the proposed modeling approach and, importantly, the construction phase. These tests could be also used to calibrate the numerical model of the steel rods proposed in the construction phase, which have been neglected for safety reasons in the finite element and rigid block analysis presented in this work.

Figure 14 shows the numbering and nesting steps and an hypothesis of the structural technological details of the joints for assembling 3D printed blocks. The number of bars depends on the width of the contact area between the blocks. We are going to improve our research by testing the method using shapes generated on a set of different parameters, such as boundary conditions, and to investigate alternative solutions for complex shapes manufacturing using blocks.

Funding Open access funding provided by Università degli Studi di Napoli Federico II within the CRUICARE Agreement.

Open Access This article is licensed under a Creative Commons Attribution 4.0 International License, which permits use, sharing, adaptation, distribution and reproduction in any medium or format, as long as you give appropriate credit to the original author(s) and the source, provide a link to the Creative Commons licence, and indicate if changes were made. The images or other third party material in this article are included in the article's Creative Commons licence, unless indicated otherwise in a credit line to the material. If material is not included in the article's Creative Commons licence and your intended use is not permitted by statutory regulation or exceeds the permitted use, you will need to obtain permission directly from the copyright holder. To view a copy of this licence, visit http:// creativecommons.org/licenses/by/4.0/.

\section{References}

Adriaenssens, Sigrid, Philippe Block, Diederick Veenendaal, and Chris Williams. 2014. Shell Structures for Architecture: Form Finding and Optimization. London: Taylor \& Francis - Routledge.

Baghdadi, Abtin, Mahmoud Heristchian and Harald Kloft. 2019. Structural Assessment of Remodelled shells of Heinz Isler. International Journal of Advanced Structural Engineering 11: 491-502.

Block, Philippe. 2021. Position: Philippe Block on Innovative Structures / Position: Philippe Block über Innovative Tragwerk. In: Architecture, Research, Building: ICD/ITKE 2010-2020 / Architektur, Forschung, Bauen: ICD/ITKE 2010-2020, eds. Achim Menges and Jan Knippers, pp. Birkhäuser.

Lachauer, Lorenz, Matthias Rippmann, and Philippe Block. 2010. Form Finding to Fabrication: A digital design process for masonry vaults. Proceedings of the International Association for Shell and Spatial Structures (IASS) Symposium, Spatial Structures-Permanent and Temporary. November 8-12 2010, Shanghai, China.

Capone, Mara and Emanuela Lanzara. 2014. Form Finding Structures: Representation methods from Analog to Digital. In: EGraFIA 2014, Revisiones del futuro, previsiones del pasado (V Congreso internacional de expresiòn gràfica - XI Congreso nacional de profesores de expresiòn gràfica en eningenierìa, arquitectura y àreas afines, 1/3 ottobre 2014 - Rosario, Argentina), eds. Héctor Carlos Lomonaco and Salvatore Barba, 486-495. CUES (editorial) and Flashbay (edicion digital). 
Cascini, Lucrezia, Raffaele Gagliardo and Francesco Portioli. 2020. LiABlock_3D: A Software Tool for Collapse Mechanism Analysis of Historic Masonry Structures. International Journal of Architectural Heritage 14(1): 75-94.

Castro Henriques, Goncalo and Juarez Moara Franco. 2020. Gridshells: integrating design with structural performance: formal and informal form finding. In: SIGraDi 2020: Transformative Design (23th Conference of the Iberoamerican Society of Digital Graphics. 16-19 November 2020). Medellín (Colombia): Escuela de Arquitectura y Diseño de la Universidad Pontificia Bolivariana.

Fallacara, Giuseppe. 2007. Verso una progettazione stereotomica. Nozioni di stereotomia, stereotomia digitale e trasformazioni topologiche: ragionamenti intorno alla costruzione della forma. Rome: Aracne editrice.

Lanzara, Emanuela. 2019. Shaping and Paneling. Superfici complesse per l'architettura e il design. Milano: FrancoAngeli.

Liu, Yang, Helmut Pottmann, Johannes Wallner, Yong Liang Yang and Wenping Wang. 2006. Geometric Modeling with Conical Meshes and Developable Surfaces. ACM Trans. Graphics 25(3): 681-689.

Portioli, Francesco Paolo Antonio. 2020. Rigid block modelling of historic masonry structures using mathematical programming: a unified formulation for non-linear time history, static pushover and limit equilibrium analysis. Bulletin of Earthquake Engineering 18(1): 211-239.

Rippmann, Matthias. 2016. Funicular Shell Design Geometric approaches to form finding and fabrication of discrete funicular structures. PhD Thesis, ETH Zurich.

Rippmann Matthias and Philippe Block. 2011a. Digital Stereotomy: Voussoir geometry for freeform masonry-like vaults informed by structural and fabrication constraints. In Proceedings of the IABSEIAAS Symposium 2011. London.

Rippmann Matthias and Philippe Block. 2011b. New Design and Fabrication Methods for Freeform Stone Vaults Based on Ruled Surfaces. In Proceedings of Design, Modeling Symposium 2011, Berlin.

Salvatore, Marta. 2012. La stereotomia scientifica in Amédée François Frézier. Prodromi della geometria descrittiva nella scienza del taglio delle pietre. Florence: Florence University Press.

Salvatore, Marta and Camillo Trevisan. 2009. Stereotomia della pietra. In: Geometria Descrittiva, Volume II: Tecniche e Applicazioni, ed. Riccardo Migliari, 484-561. Novara: Città Studi.

Sasaki, Mutsuro 2005. Flux Structure. Tokyo: Toto Publisher.

Schiftner, Alexander, Nicolas Leduc, Philippe Bompas, Niccolo Baldassini and Michael Eigensatz. 2012. Architectural Geometry from Research to Practice: The Eiffel Tower Pavilions. In: Advances in Architectural Geometry 2012, eds. Lars Hesselgren, Shrikant Sharma, Johannes Wallner, Niccolo Baldassini, Philippe Bompas, 213-228. Vienna: Springer.

Sulpizio, Concetta, Alessandra Fiore, Cristoforo Demartino, Ivo Vanzi and Bruno Briseghella. 2020. Optimal design criteria for form-finding of double-curved surfaces. Procedia Manufacturing 44(2020): 28-35.

Timoshenko, Stephen P. and S. Woinowsky-Krieger. 1959. Theory of Plates and Shells. New York: McGraw-Hill.

EN 1991. Eurocode 1: Actions on structures. CEN_European Committee for Standardization, 2003.

Publisher's Note Springer Nature remains neutral with regard to jurisdictional claims in published maps and institutional affiliations.

Mara Capone Architect, PhD in Representation and Survey of Architecture and built environment (1996), Researcher from 2005 to 2015, is Associate Professor in Drawing in the Department of Architecture (DiARC) of the Università degli Studi di Napoli Federico II. She has been involved in several research projects, including Idome (https://idome.site/about/) and "AURA - Green \& Smart Urban furniture". She has been visiting professor at: UVA Universidad de Valladolid (Spain), UFPB Universidade Federal da Paraiba (Brazil), ENSAPLV Ecole Nationale Supérieure d'Architecture de Paris la Villette (France). She has been a speaker in many international conferences. She is the author of many papers in international journals, book chapters and conference articles, mainly dealing with applied geometry, parametric design and algorithmic modelling.

Emanuela Lanzara master degree in Architecture (2010), PhD in Survey and Representation of Architecture and Environment (2015), adjunct professor of Descriptive Geometry in the Department 
of Architecture (DiARC) of the Università degli Studi di Napoli Federico II and Research Fellow for a research activity in Digital Modeling for Urban Heritage Documentation and Representation in BIM in the Department of Structures for Engineering and Architecture of the same university. She conducts international research and teaching activities on Science of Representation using advanced digital modeling and computational design techniques based on visual scripting approach. In 2018/19 and 2019/20 she taught at the Escuela Politecnica Superior, Universidad de Alicante, in the Erasmus + Teaching Staff Mobility program. Since 2011 she has collaborated on several research projects of DiARC, producing scientific publications and attending national and international conferences as author and speaker.

Francesco Paolo Antonio Portioli is a Civil Engineer with a PhD in Design (2004). He was a Postdoctoral Research Fellow in Structural Engineering at the University of Chieti-Pescara 2004-2006. He was a researcher from 2006 to 2018 and is now Professor in Structural Engineering in the Department of Architecture (DiARC) of the Università degli Studi di Napoli Federico II. He has been involved in several research projects, including "High Strength Steel in Seismic Resistant Building Frames", granted by the Research Fund for Coal and Steel, RFCS (2009-2012); COST Action C25 on "Sustainability of Constructions: Integrated Approach to Life-time Structural Engineering" , EU-COST (2006-2010); COST Action C26 "Urban Habitat Constructions under Catastrophic Events", EU-COST (2006-2010). $\mathrm{He}$ is team leader on the research topic "Masonry structures" in the RELUIS project of the Department of Italian Civil Protection Naples. He is author of many papers on international journals, book chapters and conference articles, mainly dealing with masonry and metal structures.

Francesco Flore Architect, designer for the built environment, technical consultant from 2017 for the Avellino Tribunal, is a university assistant at the Università degli Studi di Napoli Federico II. On the occasion of the master's degree thesis he worked on the parametrization of the design of a space project through the form-finding criteria. After working for a year in an architectural office, he started his path working as a digital artist, architect, and university assistant. He combines these three apparently different jobs through his 3D modelling skills. In fact, they have numerous points in common, from the mathematic soul to the creative approach. 